\title{
Author Index Vol. 15, 1994
}

(A) = Abstracts of the XXIst Meeting of the International Society for Oncodevelopmental Biology and Medicine

Abelev,G.I. 110(A) Aguirre, J. 160 Agusti,C. 318 Albert, C. 113(A) Alhava, E. 52 Alonso,D.F. 284 Alpert,E. 121(A) Andersen, J.R. 105(A) Anton, H.J. 141 Ariel, I. 123 Asanagi, M. 7

Astudillo,E. 1

Balar,D.B. 45 Balaram,P. 73 Bal de Kier Joffé, E.

284, 354 Balleari,E. 112(A) Ballesta, A.M. 1,318 Bar,J.K. 223 Bara,J. 102(A) Barak, V. 102(A), 103(A), 115(A) Bason, C. 112(A) Beauchemin, N. 107(A) Beaupain, R. 90 Beck,E.P. 103(A), 122(A) Bernades,P. 102(A) Bertolesi, G.E. 275 Biran,H. 123 Bischof Delaloye, A.

105(A) Bittl,A. 103(A), 104(A),

122 (A), 236 Blusse van Oudalblas, A.

106(A) Bohn,H. 203 Bonaparte, Y.P. de 160 Bonfrer, J.M.G. 210 Borisenko, S. 117(A)

Børmer,O.P. 185 Brown-Shimer, S.

Ill(A) Brufman, G. 115(A) Buchegger,F. 105(A)

Burde, B. 102(A), 115(A)

Cadenas, R.A. 354 Carney, W.P. Ill(A) Chaitchik, S. 188 Chandran, G.J. 73 Chariyalertsak, S.

294 Christiane, N. 116(A) Christiansen, M. 105(A) Cidre, L.L. de 275 Cilaci,S. 110(A)

Collan, Y. 345 CRES' Study Group 105 (A)

Dalen,A. de 210 Dalen,A. van 106(A) Dalifard,I. 107(A) Daniels, M. 1 Davel,L. 160 Daver,A.

107(A) Delaloye, B. 105(A) Devine,P.L. 33,337 Diament,M. 160 Di Paolo, M. 103(A) Ditter,M. 122(A) Dittrich,R. 104(A) Durán,H.A. 166

Eades-Perner, A.-M.

122(A) Eiján,A.M. 275,284,

354 Elías,C. 354 Elsässer-Beile, U. 17,

107(A), 110(A) Escribano, M.J. 102(A) Eskelinen, M. 52

Filella,X. 1,318 Fishman,P. 188 Flanagan, C.A. 82 Fomina,M. 117(A) Frängsmyr, L. 108(A)

Freeman, D.A. 203

Frenette, P.S. 247 Fujimoto, J. 311 Fujimura, S. 7 Fuks,A. 107(A)

Gallati,H. 17, 107(A) Garcia-Valdecasas, J.C.

1 Geuskens,M. 113(A) Ghadge, G.D. 255 Gharib,M. 90 Ghio,R. 112(A) Giese,G. 304 Giménez, N. 318 Gliozzo,B. 103(A) Gocze,P.M. 203 Gold, P. 107(A) Gomez, D.E. 284 Gonzalez, E. 82 Goodly, L.J. 326 Gourevitch, M. 114(A) Grau,J.J. 1 Groeneveld, E.M. 210 Groot,N.de 123 Haines, G.K. 255 Hammarström, M.-L.

108(A) Hammarström, S.

108(A) Hanazawa, Y. 7 Harasawa, S. 263 Harfozińska, A. 223 Hata,K. 230 Hauschildt, S. 304 Heering,K.J. 106(A) Hilgers,J. 114(A) Hinoda, Y. 109(A) Hirano,K. 175 Hirth,A. 122(A) Hochberg, A. 123 Hofmann, M.-C. 109(A) Høgdall,C. 105(A) Hori, M. 311 Horitsu, K. 7 Huovinen, R. 345 
Ichigo,S. 311 Iliev,I.G. 82,147 Imai,K. 109(A) Ishiguro,T. 109(A) Itoh,F. 109(A) Ivanyi,D. 210 Ivkov, N. 117(A)

Jaakkola, U.-M. 25 Jäger,W. 103(A),

104(A), 110(A),

122 (A), 236 Jasnis, M.A. 160 Jo,J. 318 Joseph, J. 318 Joseph, L. 247 Jozsa, R. 203

Kaiserling, E. 269 Kamp,G.J.van 114(A) Kannan, S. 73 Karamova, E.R. 110(A) Kastner,P. 103(A) Kato,N. 7

Kenemans, P. 114(A) Kim,K. Ill(A) Kiseleva, V.I. 110(A) Kissing, A. 110(A) Klein, S. 160 Kleist,S.von 17,107(A),

110(A), 122(A) Komoda,T. 230 Kondoh,Y. 263 Korse,C.M. 210 Koussoulakos, S. 141

Koyama, I. 230 Koyama,Y. 115(A) Kreutz,W. 304 Kupchik,H.Z. Ill(A) Kuriyama, A. 7

Kuroiwa, N. 7

Lachkar,S. 113(A) Lacy, A. de 1 Lafair,J. 103(A) Lamerz,R. 119(A)

369

Lamminen, S. 25 Lanfranco, E. Ill (A),

112(A) Lang, N. 103(A), 110(A) Larra,F. 107(A) Lathan,B. 153 Lazarevich, N.L. 110(A)

Leicach,S.R. 354 Lelouda, M. 141 Lemieux, P. 114(A) Leodolter, S. 117(A) Lévesque, A. 113

(A) Levy,M. 102(A) Levy, P. 102(A) Linde, D.L. van der

106(A) Liu,M. 120(A) Lorenzo, H.C. 113(A) Lundqvist,C. 108(A) Lustig, E.S. de 284

McGuckin, M.A. 33,

337 Mach,J.-P. 105(A) Maenner, G.A. 117(A) Mainguené, C. 90 Makarov,O. 117(A) Malhotra, P. 255 Marino, A.A. 82, 147 Marler,K,C. 82 Masuda,K. 175 Mathew,B. 73 Medl,M. 117(A)

Mercure,C. 115(A) Merimsky,O. 188 Millán,J.L. 109(A) Mincheva-Nilsson, L.

108(A) Mirimanoff, R.-O.

105 (A) Mitchell, B.S. 269 Mitomi,T. 263 Miura,M. 230 Miwa,T. 263 Molina, R. 1,318 Mori,Y. 294 Moriyama, Y. 7 Morris, D.M. 147 Müller,B. 304 Murnane,MJ. Ill(A)

Nair,M.K. 73 Nap,M. 122 (A), 236 Nishi,S. 115(A), 118(A) Nishigaki, M. 311 Nishihira,J.

115(A) Nisman,B. 102(A),

103(A), 115(A) Nørgaard-Pedersen, B.

105 (A) Novell, F. 1 Nunez, E.A. 63, 119(A)

Ogawa, M. 7 Ogoshi,K. 263 Ogris,E. 117(A) Ohkura,H. 294 Ohmori,A. 120(A) Onsrud,M. 361

Oomen, L.C.J.M. 210

Pagé,B. 116(A) Pagé,M. 113(A), 114(A),

115(A), 116(A) Palapelas, V. 110(A) Palmieri, M.A. 166 Papa, V. 103(A) Paquet,A. 113(A)

Partanen, K. 52 Pasanen, P.A. 52 Patel,P.S. 45 Penttilä, I. 52 Peretz,T. 102(A) Perron, M.-J.

116(A) Peters-Engel, C. 117(A) Pezzino,V. 103(A) Pikkarainen, P. 52 Pillai,M.R. 73 Pique,

J.M. 1 Pohl,A.L. 117(A) Poverenny, A.M. 110(A) Prokopenko, P. 117(A) Puricelli, L. 284, 354

Putten, H. van der

122(A) Pyykkö,K. 25

Quin,R.J. 337

Radosevich, J.A. 255 Ramm, L.E. 33 Rawal,G.N. 45 Rey,B.M. de 166 Rogers, S. 147

Roisman,I. 102(A), 103(A), 115(A)

Rosa,F. Ill(A), 112(A)

Russo,P. 103(A)

Sakagishi,Y. 230 Sakai,H. 230 Sakai,M. 115(A),

118(A) Sato,K. 7 
Schindler, S. 104(A) Schroy,P.C. Ill(A) Schulte Mönting, J. 17 Schumacher, U. 269 Schwalke, M.A. 82,147 Schweiger, Ch.R. 117(A) Sekiya,C. 120(A) Senoda,A. 109(A) Severin, T. 304 Shabana, A. 361 Shani,A. 123 Shoenfeld,Y. 188 Shuster,J.S. 247 Siegal, G.P. 326 Singh, R.K. 326 Sobis,H. 118(A) Söderström, K.-O. 25 Spinelli,O. 284 Stanners, C.P. 107(A) Stieber,P. 119(A) Stöckelhuber, B. 119(A) Sugano,K. 294 Sumida,C. 119(A) Szabo,D.G. 203

Taga,H. 120(A) Tagirova, A.K. 120(A) Tajima, T. 263 Takagishi, Y. 175 Takahashi,K. 175 Taketa,K. 120(A) Tamaya, T. 311 Tamboise, A. 90 Tamboise, E. 90 Tani,N. 263 Tatamnov, Y.S. 117(A), 120(A), 121(A) Teglund,S. 108(A) Terentjev, A.A. 120(A),

121 (A) Thierauf,P. 104(A) Thimmappya, B. 255

Thirlwell, M.P. 247 Thompson, J. 122(A) Thomson, D.M.P. 247 Trudeau, M. 247 Tsuchida,Y. 109(A) Tuchais,C. 107(A) Tuchais,E. 107(A)

Uriel, J. 113(A)

Vandeputte, M. 118(A) Van Mensdorff-Pouilly, S.

114(A) Verdière-Sahuque, M.

113(A) Veromann, S. 135 Verstaeten, A. 114(A) Verstuyf,A. 118(A) Vidal, M.d.C.C. 284

Vilgrain,V. 102(A) Vogel,C.-A. 105(A) Voitot,H. 102(A)

Wang,M.H. 326 Wang,W. 121(A) Ward,B.G. 33,337 Weber, W. 17 Weigang,B. 122(A), 236 Weisser,H. 153 Wetterauer, U. 107(A) Wild,E.D. 17 Wildt,L. 103(A), 104(A) Witzel,J.G 153 Wolf,B. 304

Yachi,A. 109(A) Yamagishi, F. 230 Yamamoto,H. 109(A) Yecheskel, G. 188 Yoshimoto,M. 109(A)

Zhou, G.-Q. 108(A) Zimmermann, W.

122(A)

370

Author Index 